\title{
Myeloid sarcoma in the breast in a patient with acute myeloid leukemia: a case report
}

\author{
Ana Lucia Kefalas Oliveira ${ }^{\circ}$, July Helen Marinho de Almeida1* ${ }^{\circ}$,

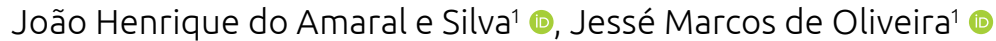

\section{ABSTRACT}

Myeloid sarcoma infiltration into the breast of patients with acute myeloid leukemia is rare. The present study reports the case of a 56-year-old woman diagnosed with AML and an incidental finding of a breast tumor. The nodule biopsy raised the suspicion of invasive lobular carcinoma and poorly differentiated angiosarcoma. Subsequent immunohistochemical study concluded the diagnosis of myeloid sarcoma. The varied image presentations, the lack of knowledge of clinical data and complementary propaedeutics, and the histopathological similarity with certain primary breast lesions make it difficult to discover secondary infiltration by myeloid sarcoma in this unusual site.

KEYWORDS: breast neoplasms; sarcoma, myeloid; leukemia.

\section{INTRODUCTION}

Myeloid sarcoma (MS) is the tumor form of acute myeloid leukemia (AML), consisting of a collection of myeloid blasts in an anatomical site other than the bone marrow. It is addressed by several names, including myeloblastoma, monocytic sarcoma and chloroma. Other synonyms are granulocytic sarcoma and extramedullary myeloid tumor ${ }^{1}$. Although it can affect any region of the body, its presentation in the breast is extremely uncommon, having been, until 2005, only 67 cases recorded in the literature ${ }^{2}$, with additional episodes reported sporadically until recently ${ }^{3}$. Skin, lymph nodes, gastrointestinal tract, bone, soft tissues and testicles are the most frequent sites of involvement ${ }^{4}$. MS can be found isolated in about a quarter of cases, or during the course of AML, chronic myeloid leukemia, myelodysplastic syndrome or other myeloproliferative disorders ${ }^{3}$. MS occurs with an incidence of $2 \%$ to $14 \%$ in AML ${ }^{5}$. The age of onset varies from 29 to 72 years, mean age of 42 years ${ }^{1}$. It is difficult to define typical characteristics of the affected patients, so the diagnosis can only be confirmed through pathological examination with immunohistochemistry.

\section{CASE REPORT}

A 56-year-old female patient presented at the emergency unit complaining of adynamia, moderate dyspnea, hyporexia, and weight loss within the past two months, with symptomatic worsening in the last 15 days. She carried recent tests that revealed significant anemia, thrombocytopenia, and leukocytosis, and was admitted to our institution for investigation. On physical examination, she was pale, sarcopenic, dehydrated, and had multiple lymph node enlargements. She was diagnosed with AML subtype M4, using bone marrow aspirate, and induction chemotherapy with cytarabine was started six days after admission.

Chest tomography performed to assess respiratory distress revealed an incidental finding of a nodule in the right breast and axillary lymph node enlargement. Mammography (Figure 1) revealed a nodule in the right breast measuring $2 \mathrm{~cm}$, located at the junction of the lateral quadrants, oval, with indistinct margins, being categorized as a suspected lesion of malignancy. Complementary ultrasonography (Figure 2) confirmed the suspicious findings, identifying an oval nodule parallel to the skin, circumscribed, heterogeneous, with slight posterior acoustic reinforcement, without flow to the color Doppler study, at the union of the lateral quadrants on the right, at 9 o'clock, $4 \mathrm{~cm}$ from the papilla, and measuring $2 \times 1.4 \times 1.8 \mathrm{~cm}$. A thick needle biopsy revealed breast tissue infiltrated by round, diseased cells, with a high nucleus-cytoplasm ratio. In the absence of clinical and laboratory information, and due to the probabilities, the hypotheses of invasive lobular carcinoma and poorly differentiated angiosarcoma were raised. However, an immunohistochemical

${ }^{1}$ Hospital das Clínicas da Universidade Federal do Triângulo Mineiro - Uberaba (MG), Brazil.

*Corresponding author: july_helen3@hotmail.com

Conflict of interests: nothing to declare.

Received on: 11/29/2020. Accepted on: 03/03/2021 
study ruled out these hypotheses by revealing a negative result for cytokeratins 7 and AE1/AE2.

The patient's clinical weakness postponed the surgical approach to breast injury, which was followed up with imaging tests until conditions favored invasive treatment. A new mammogram (Figure 3), performed seven months following the first, after three complete chemotherapy cycles, no longer showed the nodule, which on ultrasound showed a significant reduction in tumor mass. Leukemic infiltration in the breast became the main clinical

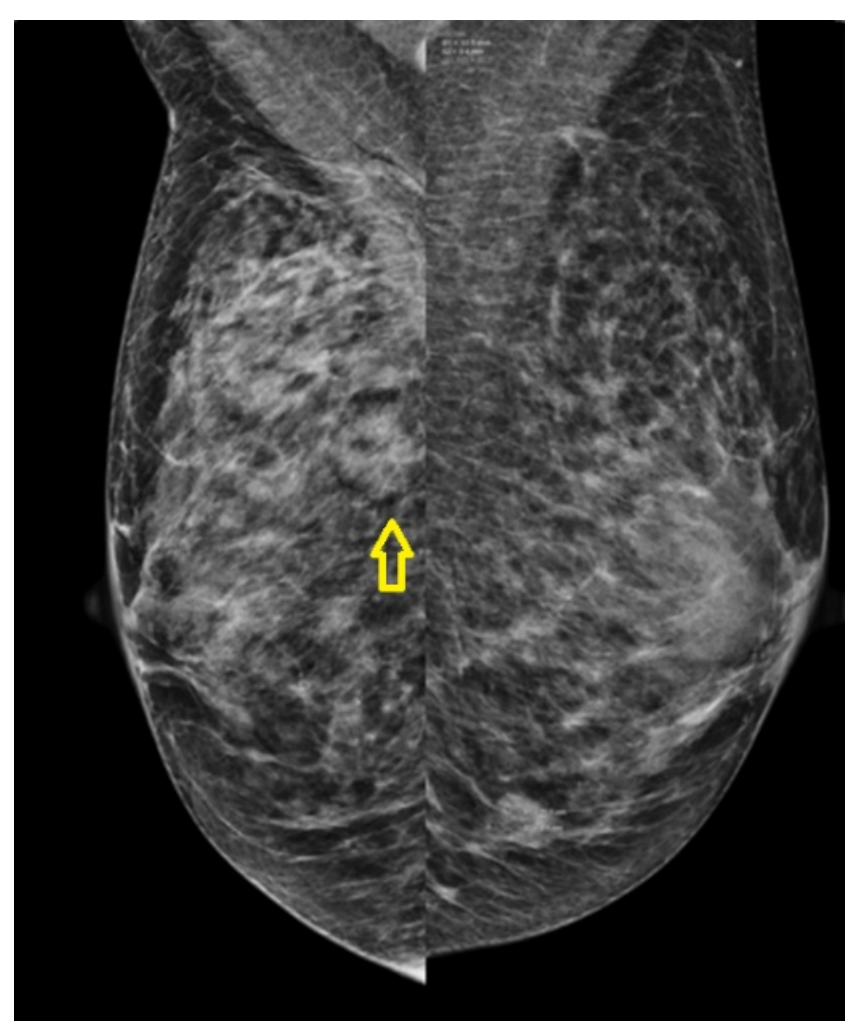

Figure 1. Mammography in oblique mediolateral view showing a nodule in the right breast, measuring $2 \mathrm{~cm}$, at the junction of the lateral quadrants, oval, with indistinct margins (BI-RADS 4A).

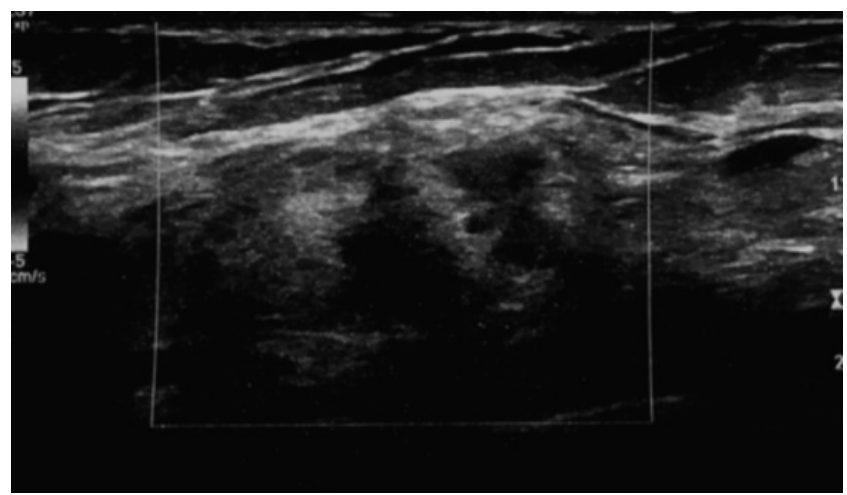

Figure 2. Ultrasonography showing an oval nodule, parallel to the skin, circumscribed, heterogeneous, without flow to the color Doppler study, at the union of the lateral quadrants, at $9 \mathrm{am}, 4 \mathrm{~cm}$ apart from the papilla, measuring $2 \times 1.4 \times 1.8 \mathrm{~cm}$. suspicion, considering the behavior of the tumor in the face of chemotherapy directed at leukemia and the inconsistent diagnoses between anatomopathological and immunohistochemical studies.

Nine months after the diagnosis, the patient underwent an open excisional biopsy, and the examination of the surgical specimen showed sparse foci of remaining neoplasia. A new immunohistochemical study, in the light of clinical information, resulted in positive tumor cells for the CD34, CD45, lysozyme, CD15, and myeloperoxidase markers. Thus, the diagnosis of breast infiltration by MS was confirmed. Ten months after diagnosis, and after four cycles of chemotherapy, the patient died as a result of complications from the underlying disease.

\section{DISCUSSION}

MS can occur in three clinical contexts: simultaneously with blood and bone marrow involvement, as in the case of our patient; as isolated recurrence of AML; and prior to the manifestations of systemic leukemia ${ }^{6}$. Even in patients with bone marrow invasion, breast MS is quite uncommon. Patients with breast MS have mainly a painless mass, without inversion or nipple secretion? In the case studied, the patient did not present evident symptoms. However, previous studies report both asymptomatic presentation and presentation of painful palpable nodulation ${ }^{7}$. Therefore,

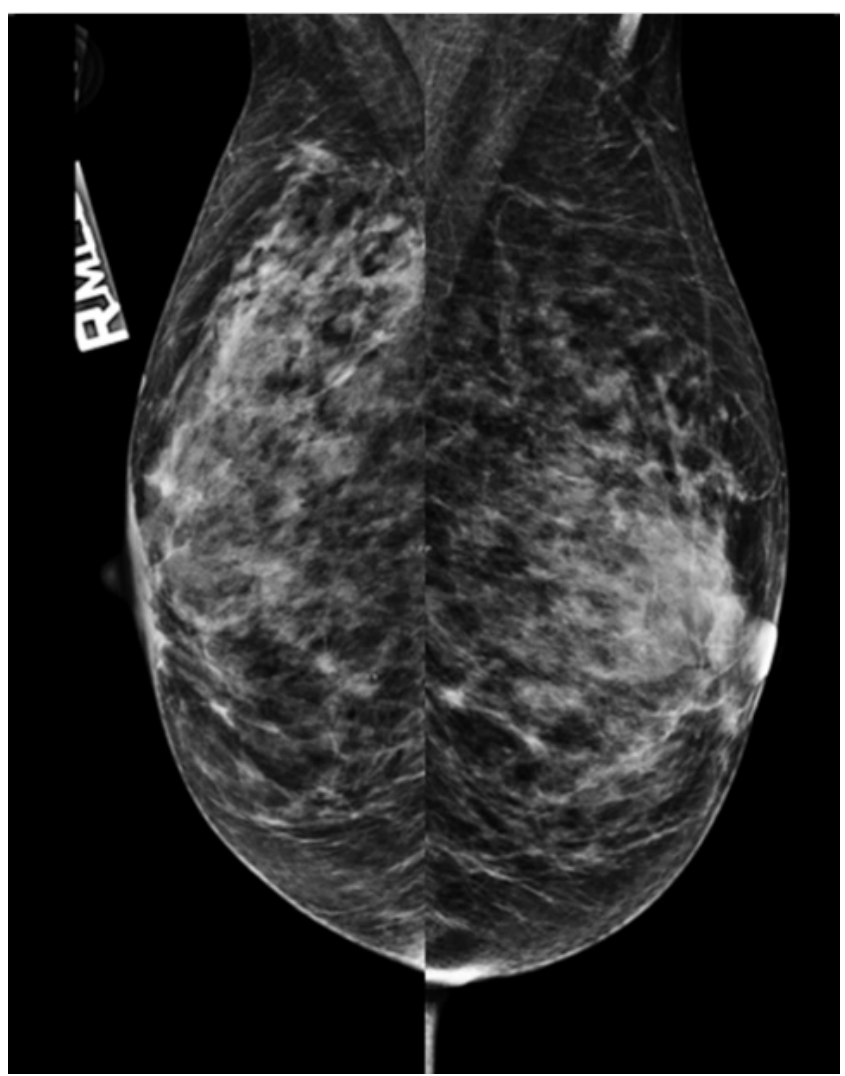

Figure 3. Mammography performed after 3 cycles of chemotherapy no longer demonstrated the nodule. 
it is difficult to define typical clinical presentations of the tumor. The morphological, imaging, and histological characteristics are as variable as the clinical presentation, of difficult diagnosis, especially if it is of low suspicion. Mammography shows that breast leukemias have three mammographic patterns: breast masses, architectural distortions, and no abnormalities. Most breast masses are hyperdense, have a rounded shape and microlobulated margins, and occasionally accompany internal microcalcifications. On ultrasound, they usually present as solitary or multiple masses that tend to be homogeneously hypoechoic with microlobulated or indistinct margins ${ }^{2}$. The immunohistochemical study is extremely useful in recognizing MS, the most specific markers of myeloid differentiation being myeloperoxidase and lysozyme, both positive in this case. The levels of myeloperoxidase positive cell expression in MS tend to be between 66 and $83.6 \%^{6}$. The most common differential diagnoses include invasive lobular carcinoma, non-Hodgkin's lymphoma or even nonneoplastic conditions, such as inflammation and extramedullary hematopoiesis ${ }^{8}$. In the reported case, the diagnosis of invasive lobular carcinoma was the first to be considered.

The treatment modalities recorded in the literature include surgical excision, radiotherapy, and chemotherapy and depend on the patient's clinical conditions, the size of the tumor, and the systemic response. However, most studies have concluded that all patients with MS should receive mastectomy or breast sectorectomy combined with standard systemic chemotherapy, and overall survival appears to be longer in patients treated with chemotherapy compared to those who do not receive it. Although the patient in the case presented has died, due to previous clinical weakness, it is important to note that the response of the breast tumor to chemotherapy was quite significant, since it was no longer identified in the follow-up mammography and had a significant reduction demonstrated on ultrasound.

\section{CONCLUSION}

The case presented here shows the importance of the clinicalpathological correlation and maintenance of high diagnostic suspicion for MS in patients with AML, although morphological or histological characteristics suggest other conditions. In the case of the presented patient, the diagnosis of AML helped to consider the diagnostic possibility of MS and, consequently, contributed to a satisfactory mammary tumor regression. The rarity of breast involvement by this type of tumor means that most of the information available on its behavior and its manifestations is obtained from case reports and small retrospective studies. Its extremely variable presentation makes diagnosis difficult through imaging exams, requiring the use of all the resources necessary for anatomopathological and immunohistochemical diagnosis.

\section{AUTHORS' CONTRIBUTION}

A.L.K.O.: conceptualization, investigation, methodology, project administration, supervision, validation.

J.H.M.A.: methodology, research, writing - original draft, writing - review \& editing).

J.H.A.S.: writing - review \& editing, validation.

J.M.O.: writing - review \& editing.

\section{REFERENCES}

1. Nalwa A, Nath D, Suri V, Jamaluddin MA, Srivastava A. Myeloid sarcoma of the breast in an aleukemic patient: a rare entity in an uncommon location. Malays J Pathol. 2015;37(1):63-6.

2. Kim SJ, Kim WG. Sonographic Features of a Myeloid Sarcoma of the Breast as a Relapse of Acute Myeloid Leukemia After Stem-Cell Transplantation: A Case Report. Am J Case Rep. 2019;20:612-9. https://doi.org/10.12659/AJCR.915453

3. Zhai J, Kong X, Yang X, Gao J, Xuan L, Wang X, et al. An uncommon granulocytic sarcoma of the breast: a case report and literature review. Onco Targets Ther. 2018;11:3685-90. https://doi.org/10.2147/OTT.S149149

4. Gomaa W, Ghanim A, Emam E, Bayoumi K, Ghanim A. Primary Myeloid Sarcoma of the Breast: A Case Report and Review of Literature. J Microsc Ultrastruct. 2018;6(4):212-4. https://doi.org/10.4103\%2FJMAU.JMAU_15_18
5. Huang XE, Li YJ, Zhou XD. Granulocytic sarcoma of the breast: A case report. Oncol Lett. 2015;10(4):2447-9. https://doi. org/10.3892/ol.2015.3532

6. Wu HY, Liu L, Gu L, Luo YH. Clinical characteristics and management of primary granulocytic sarcoma of the breast: A case report. Medicine (Baltimore). 2019;98(35):e16648. https://doi.org/10.1097/MD.0000000000016648

7. Sharma A, Das AK, Pal S, Bhattacharyya S. Fine-needle aspiration cytology of granulocytic sarcoma presenting as a breast lump Report of a rare case with a comprehensive literature search.J Lab Physicians. 2018;10(1):113-5.https://doi.org/10.4103/JLP.JLP_114_17

8. Fernandes Vieira V, Vo QD, Bouquet de la Jolinière J, Khomsi F, Feki A, Hoogewoud HM. Granulocytic Sarcoma Presenting as a Palpable Breast Lump. Front Surg. 2017;3:67. https://doi. org/10.3389/fsurg.2016.00067 